\title{
Residuation Properties and Weakly Primary Elements in Lattice Modules
}

\author{
C. S. Manjarekar ${ }^{1}$ and U. N. Kandale ${ }^{2}$ \\ ${ }^{1}$ Department of Mathematics, Shivaji University, Kolhapur, India \\ ${ }^{2}$ General Engineering Department, Sharad Institute of Technology College of Engineering, Yadrav, Ichalkaranji, India
}

Correspondence should be addressed to U. N. Kandale; ujwalabiraje@gmail.com

Received 7 July 2014; Accepted 14 November 2014; Published 27 November 2014

Academic Editor: Hong You

Copyright ( 2014 C. S. Manjarekar and U. N. Kandale. This is an open access article distributed under the Creative Commons Attribution License, which permits unrestricted use, distribution, and reproduction in any medium, provided the original work is properly cited.

We obtain some elementary residuation properties in lattice modules and obtain a relation between a weakly primary element in a lattice module $M$ and weakly prime element of a multiplicative lattice $L$.

\section{Introduction}

A multiplicative lattice $L$ is a complete lattice provided with commutative, associative, and join distributive multiplication in which the largest element $I$ acts as a multiplicative identity. An element $a \in L$ is called proper if $a<1$. A proper element $p$ of $L$ is said to be prime if $a b \leq p$ implies $a \leq p$ or $b \leq p$. If $a \in L, b \in L,(a: b)$ is the join of all elements $c$ in $L$ such that $c b \leq a$. A proper element $p$ of $L$ is said to be primary if $a b \leq p$ implies $a \leq p$ or $b^{n} \leq p$ for some positive integer $n$. If $a \in L$, the radical of a is denoted by $\sqrt{a}=\vee\left\{x \in L \mid x^{n} \leqslant a, n \in Z_{+}\right\}$. An element $a \in L$ is called compact if $a \leqslant \vee_{\alpha} b_{\alpha}$ implies $a \leqslant b_{\alpha 1} \vee b_{\alpha 2} \vee \cdots \vee b_{\alpha n}$ for some finite subset $\left\{\alpha_{1}, \alpha_{2}, \ldots, \alpha_{n}\right\}$. Throughout this paper, $L$ denotes a compactly generated multiplicative lattice with 1 compact and every finite product of compact elements is compact. We will denote by $L_{*}$ the set compact elements of $L$. A nonempty subset $F$ of $L_{*}^{*}$ is called a filter of $L_{*}$ if the following conditions are satisfied:

(1) $x, y \in F$ implies $x y \in F$;

(2) $x \in F, x \leqslant y$ implies $y \in F$.

Let $F\left(L_{*}\right)$ denote the set of all filters of $L$. For a nonempty subset $\left\{F_{\alpha}\right\} \subseteq F\left(L_{*}\right)$, define $\mathbb{E} F_{\alpha}=\left\{x \in L_{*} \mid x \geq f_{1} f_{2} \cdots f_{n} \in\right.$ $F_{\alpha_{i}}$, for some $\left.i=1,2, \ldots, n\right\}$. Then it is observed that $F\left(L_{*}\right)=$ $\left\langle F\left(L_{*}\right), \mathbb{E}, \cap\right\rangle$ is a complete distributive lattice with $\mathbb{E}$ as the supremum and the set theoretic $\bigcap$ as the infimum. For $a \in L_{*}$ the smallest filter containing a is denoted by $[a]$ and it is given by $[a)=\left\{x \in L * \mid x \geq a^{n}\right.$ for some nonnegative integer $\left.n\right\}$. For a filter $F \in F\left(L_{*}\right)$ we denote $0_{F}=\vee\left\{x \in L_{*} \mid x s=0\right.$, for $s \in F\}$.

Let $M$ be a complete lattice and $L$ a multiplicative lattice. Then $M$ is called $L$-module or module over $L$ if there is a multiplication between elements of $L$ and $M$ written as $a B$ where $a \in L$ and $B \in M$ which satisfies the following properties:

(1) $\left(\vee_{\alpha} a_{\alpha}\right) A=\vee_{\alpha} a_{\alpha} A, \forall a_{\alpha} \in L, A \in M$;

(2) $a\left(\vee_{\alpha} A_{\alpha}\right)=\vee_{\alpha} a A_{\alpha}, \forall a \in L, A_{\alpha} \in M$;

(3) $(a b) A=a(b A), \forall a, b \in L, A \in M$;

(4) $I B=B$;

(5) $O B=O_{M}$, for all $a, a_{\alpha}, b \in L$ and $A, A_{\alpha} \in M$, where $I$ is the supremum of $L$ and $O$ is the infimum of $L$. We denote by $0_{M}$ and $I_{M}$ the least element and the greatest element of $M$. Elements of $L$ will generally be denoted by $a, b, c, \ldots$ and elements of $M$ will generally be denoted by $A, B, C, \ldots$.

Let $M$ be an $L$-module. If $N \in M$ and $a \in L$ then $(N$ : $a)=\vee\{X \in M \mid a X \leqslant N\}$. If $A, B \in M$, then $(A: B)=\vee\{x \in$ $L \mid x B \leqslant A\}$. An $L$-module $M$ is called a multiplication $L$ module if for every element $N \in M$ there exists an element $a \in L$ such that $N=a I_{M}$. In this paper a lattice module $M$ will be a multiplication lattice module. A proper element $N$ of 
$M$ is said to be prime if $a X \leqslant N$ implies $X \leqslant N$ or $a I_{M} \leqslant N$; that is, $a \leqslant\left(N: I_{M}\right)$ for every $a \in L, X \in M$. If $N$ is a prime element of $M$ then $\left(N: I_{M}\right)$ is prime element of $L[1]$. An element $N<I_{M}$ in $M$ is said to be primary if $a X \leqslant N$ implies $X \leqslant N$ or $a^{n} I_{M} \leqslant N$; that is, $a^{n} \leqslant\left(N: I_{M}\right)$ for some integer $n$. An element $N$ of $M$ is called a radical element if $\left(N: I_{M}\right)=\sqrt{\left(N: I_{M}\right)}$.

\section{Residuation Properties}

We prove some elementary properties of residuation. Such results are obtained by Anderson and others [2] for compactly generated multiplicative lattices.

Theorem 1. Let $L$ be a multiplicative lattice and $M$ a multiplication lattice module over L. For $x, y \in L$ and $Z, A, B \in M$, where $\left(0_{M}: I_{M}\right)$ is a radical element, one has the following identities:

(i) $x \leqslant y$ implies $\left(0_{M}: y\right) \leqslant\left(0_{M}: x\right)$ and $0_{M}:\left(0_{M}:\right.$ $x) \leqslant 0_{M}:\left(0_{M}: y\right)$;

(ii) $x \leqslant 0_{M}:\left(0_{M}: x\right)$;

(iii) $0_{M}:\left[0_{M}:\left(0_{M}: x\right)\right]=\left(0_{M}: x\right)$;

(iv) $\left(0_{M}: x\right)=\left(0_{m}: x^{n}\right)$ for every $n \in Z_{+}$;

(v) $0_{M}:\left(0_{M}: x\right) \wedge 0_{M}:\left(0_{M}: y\right)=0_{M}:\left(0_{M}: x y\right)=$ $0_{M}:\left[0_{M}:(x \wedge y)\right]$

(vi) $\left(0_{M}: a\right)=0_{M}$ implies $\left(0_{M}: a^{n}\right)=0$ for every $n \in Z_{+}$;

(vii) $x \vee y=1$ implies $\left(0_{M}: x\right) \vee\left(0_{M}: y\right)=0_{M}:(x \wedge y)=$ $0_{M}: x y$;

(viii) For $Z$ in $M, Z \leqslant 0_{M}:\left(0_{M}: Z\right)$;

(ix) $A \leqslant B$ implies $\left(0_{M}: B\right) \leqslant\left(0_{M}: A\right)$;

(x) $0_{M}:\left[0_{M}:\left(0_{M}: A\right)\right]=0_{M}: A$;

(xi) $0_{M}: x I_{M}=0_{M}: x^{n} I_{M}$ for some positive integer $n$.

Proof. (i) We have $\left(0_{M}: y\right)=\vee\left\{A \in M \mid y A=0_{M}\right\}$. As $x \leqslant$ $y, x A \leqslant y A$, so $y A=0_{M}$ implies $x A=0_{M}$. This shows that $\left(0_{M}: y\right) \leqslant\left(0_{M}: x\right)$. Next part $0_{M}:\left(0_{M}: x\right) \leqslant 0_{M}:\left(0_{M}: y\right)$ is obvious.

(ii) We have $x\left(0_{M}: x\right)=x\left\{\vee\left\{A \mid x A=0_{M}\right\}\right\}=\vee\{x A \mid$ $\left.x A=O_{M}\right\}=0_{M}$. This implies that $x \leqslant 0_{M}:\left(0_{M}: x\right)$.

(iii) We have, by (ii), $x \leqslant 0_{M}:\left(0_{M}: x\right)$. So by (i), $0_{M}$ : $\left[0_{M}:\left(0_{M}: x\right)\right] \leqslant\left(0_{M}: x\right)$. If $A \in M, A\left(0_{M}: A\right)=\vee\{x A \mid$ $\left.x A=0_{M}\right\}=0_{M}$. So, $A \leqslant 0_{M}:\left(0_{M}: A\right)$. Hence, $\left(0_{M}:\right.$ $x) \in M$ implies $\left(0_{M}: x\right) \leqslant 0_{M}:\left[0_{M}:\left(0_{M}: x\right)\right]$. Therefore, $0_{M}:\left[0_{M}:\left(0_{M}: x\right)\right]=\left(0_{M}: x\right)$.

(iv) We have $\left(0_{M}: x\right)=\vee\left\{A \in M \mid x A \leqslant 0_{M}\right\}$. As $x^{n} \leqslant x$, we have $\left(0_{M}: x\right) \leqslant\left(0_{M}: x^{n}\right)$ for every $n \in Z_{+}$. Let $Z=\left(0_{M}: x^{n}\right)$ where $Z \in M$. As $M$ is a multiplication lattice module $Z=a I_{M}$ for some $a \in L$. So $x^{n} a^{n} I_{M} \leqslant x^{n} a I_{M}=0_{M}$. Hence $x a \leqslant \sqrt{\left(0_{M}: I_{M}\right)}=\left(0_{M}: I_{M}\right)$. So $x a I_{M} \leqslant 0_{M}$; that is, $Z \leqslant\left(0_{M}: x\right)$, and hence $\left(0_{M}: x\right)=\left(0_{M}: x^{n}\right)$ for each positive integer $n$.

(v) Note that $x y \leqslant x, y$. Therefore by (i), $0_{M}:\left(0_{M}: x y\right) \leqslant$ $0_{M}:\left(0_{M}: x\right) \wedge 0_{M}:\left(0_{M}: y\right)$. Let $z=0_{M}:\left(0_{M}: x\right) \wedge 0_{M}:$ $\left(0_{M}: y\right)$. Now we have $z \leqslant 0_{M}:\left(0_{M}: x\right)$ and $z \leqslant 0_{M}:\left(0_{M}:\right.$ $y)$. Then, $z\left(0_{M}: x\right)=0_{M}$ and $z\left(0_{M}: y\right)=0_{M}$. This implies that $\left(0_{M}: x\right) \leqslant\left(0_{M}: z\right),\left(0_{M}: y\right) \leqslant\left(0_{M}: z\right)$. Therefore, we have $\left(0_{M}: x y\right)=\left(0_{M}: x\right): y \leqslant\left(0_{M}: z\right): y=\left(0_{M}: y z\right)$ [3]. Hence, $\left(0_{M}: x y\right) \leqslant\left(0_{M}: y\right): z \leqslant\left(0_{M}: z\right): z=$ $\left(0_{M}: z^{2}\right)=\left(0_{M}: z\right)$, by (iv). That is, $z\left(0_{M}: x y\right)=0_{M}$ or $z \leqslant 0_{M}:\left(0_{M}: x y\right)$. Consequently, $0_{M}:\left(0_{M}: x y\right)=0_{M}:$ $\left(0_{M}: x\right) \wedge 0_{M}:\left(0_{M}: y\right)$. Note that $x \wedge y \leqslant x, y$. Then by (i), we have $0_{M}:\left(0_{M}:(x \wedge y)\right) \leqslant 0_{M}:\left(0_{M}: x\right) \wedge 0_{M}:\left(0_{M}: y\right)$. Let $z=0_{M}:\left(0_{M}: x\right) \wedge 0_{M}:\left(0_{M}: y\right)$. We have $0_{M}:(x \wedge y) \leqslant$ $0_{M}: x y \leqslant 0_{M}: z^{2}=0_{M}: z$, by (iv). This shows that $z\left[0_{M}:\right.$ $(x \wedge y)] \leqslant 0_{M}$. Thus, $z \leqslant 0_{M}:\left[0_{M}:(x \wedge y)\right]$. Consquently, $0_{M}:\left[0_{M}:(x \wedge y)\right]=0_{M}:\left(0_{M}: x\right) \wedge 0_{M}:\left(0_{M}: y\right)$.

(vi) Assume that $\left(0_{M}: a\right)=0_{M}$. We have, by (iv), $\left(0_{M}\right.$ : $a)=\left(0_{M}: a^{n}\right)$ for every $n \in Z_{+}$. But $\left(0_{M}: a\right)=0_{M}$. Thus, $\left(0_{M}: a^{n}\right)=0_{M}$ for every $n \in Z_{+}$.

(vii) Assume that $x \vee y=1$. As $x \wedge y \leqslant x$, $y$, we have $\left(0_{M}: x\right) \leqslant\left(0_{M}:(x \wedge y)\right),\left(0_{M}: y\right) \leqslant\left(0_{M}:(x \wedge y)\right)$. Therefore, $\left(0_{M}: x\right) \vee\left(0_{M}: y\right) \leqslant 0_{M}:(x \wedge y)$. Let $Z$ in $M$ be such that $Z \leqslant 0_{M}:(x \wedge y)$. Then, $Z x y \leqslant Z(x \wedge y) \leqslant 0_{M}$. This gives $Z x \leqslant\left(0_{M}: y\right)$ and $Z y \leqslant\left(0_{M}: x\right)$. Then we have, for $Z$ in $M, Z=Z .1=Z(x \vee y)=Z x \vee Z y \leqslant\left(0_{M}: x\right) \vee\left(0_{M}: y\right)$. Consequently, $0_{M}:(x \wedge y)=\left(0_{M}: x\right) \vee\left(0_{M}: y\right)$ For the next part, $x y \leqslant x, y$ where $x, y \in L$. We have $\left(0_{M}: x\right) \leqslant\left(0_{M}: x y\right)$, $\left(0_{M}: y\right) \leqslant\left(0_{M}: x y\right)$. Therefore, we get $\left(0_{M}: x\right) \vee\left(0_{M}: y\right) \leqslant$ $\left(0_{M}: x y\right)$. Let $Z$ be an element of $M$ such that $Z \leqslant 0_{M}: x y$. Then, $x y Z \leqslant 0_{M}$. This gives $x Z \leqslant\left(0_{M}: y\right)$ and $y Z \leqslant\left(0_{M}\right.$ : $x)$. Then we have $Z=1 . Z=(x \vee y) Z=x Z \vee y Z \leqslant\left(0_{M}\right.$ : $x) \vee\left(0_{M}: y\right)$. Consequently, $0_{M}:(x y)=\left(0_{M}: x\right) \vee\left(0_{M}: y\right)$.

(viii) We have, for $Z$ in $M, Z\left(0_{M}: Z\right)=Z[\vee\{a \in L$ । $\left.\left.a Z=0_{M}\right\}\right]=\vee\left\{a Z \mid a \in L\right.$ and $\left.a Z=0_{M}\right\}$. This implies that $Z\left(0_{M}: Z\right)=0_{M}$. This shows that, for $Z$ in $M, Z \leqslant 0_{M}:\left(0_{M}:\right.$ $Z)$.

(ix) Let $A \leqslant B$. We have $x \leqslant\left(0_{M}: B\right)$ implies $x B=0_{M}$. $A \leqslant B$ implies $x A \leqslant x B=0_{M}$. So, $x \leqslant\left(0_{M}: A\right)$. Therefore, $\left(0_{M}: B\right) \leqslant\left(0_{M}: A\right)$.

(x) As $\left(0_{M}: A\right) \in L$, by (ii), we get $\left(0_{M}: A\right) \leqslant 0_{M}:\left[0_{M}\right.$ : $\left.\left(0_{M}: A\right)\right]$. But $A \leqslant 0_{M}:\left(0_{M}: A\right)$. Therefore, $\left(0_{M}: A\right) \geq 0_{M}$ : $\left[0_{M}:\left(0_{M}: A\right)\right]$. Thus, $0_{M}:\left[0_{M}:\left(0_{M}: A\right)\right]=\left(0_{M}: A\right)$.

(xi) As $x^{n} \leqslant x$, we have $x^{n} I_{M} \leqslant x I_{M}$. So $\left(0_{M}: x I_{M}\right) \leqslant$ $\left(0_{M}: x^{n} I_{M}\right)$. Let $z=\left(0_{M}: x^{n} I_{M}\right)$ where $z \in L$. Then $z x^{n} I_{M} \leqslant$ $0_{M}$; that is, $x^{n} z I_{M}=0_{M}$. Therefore, $x^{n} z^{n} I_{M}=(x z)^{n} I_{M} \leqslant$ $x^{n} z I_{M}=0_{M}$. Hence, $x z \leqslant \sqrt{\left(0_{M}: I_{M}\right)}=\left(0_{M}: I_{M}\right)$, since $0_{M}$ is a radical element. This implies that $z \leqslant\left(0_{M}: x I_{M}\right)$. Consequently, $\left(0_{M}: x^{n} I_{M}\right) \leqslant\left(0_{M}: x I_{M}\right)$. Hence, $\left(0_{M}\right.$ : $\left.x I_{M}\right)=\left(0_{M}: x^{n} I_{M}\right)$.

Let $M_{*}$ denote the set of compact elements of $M$. We define $0_{F M}=\vee\left\{X \in M_{*} \mid s X=0_{M}\right.$ for some $\left.s \in F\right\}$. Such concept is defined for multiplicative lattices by Anderson and others [2].

We obtain the necessary and sufficient condition for an element of a lattice module $M$ to be contained in $0_{F M}$.

Theorem 2. Let $F \subseteq L$ be a filter of $F\left(L_{*}\right)$ and let $X$ be a compact element of $M$. Then $X \leqslant 0_{F M}$ if and only if $s X=0_{M}$ for some $s \in F$.

Proof. As $X$ is compact element and $X \leqslant 0_{F M}$, there exist finite number of elements $X_{1}, X_{2}, \ldots, X_{n}$ such that $X \leqslant$ $X_{1} \vee X_{2} \vee \cdots \vee X_{n}$ where $s_{i} X_{i}=0_{M}$ for some $s_{i} \in F$. Let 
$s=s_{1} s_{2} \cdots s_{n}$. Then by hypothesis, finite product of compact elements is compact, and hence, $s$ is a compact element. As $F$ is a filter, $s \in F$. Also, $s \leqslant s_{i}$ for each $i$. Hence, we have $s X \leqslant s\left(X_{1} \vee X_{2} \vee \cdots \vee X_{n}\right)=s X_{1} \vee \cdots \vee s X_{n} \leqslant s_{1} X_{1} \vee$ $s_{2} X_{2} \vee \cdots \vee s_{n} X_{n}=0_{M}$. Conversely, suppose $s X=0_{M}$ for some $s \in F$. Hence, $X \leqslant 0_{F M}$ obviously.

Now we obtain an equivalent formulation for $0_{F M}$.

Theorem 3. For $F \in F\left(L_{*}\right), 0_{F M}=\vee\left\{\left(0_{M}: x\right) \mid x \in F\right\}$.

Proof. Let $A=\vee\left\{\left(0_{M}: x\right) \mid x \in F\right\}$. Let $B$ be a compact element of $M$ such that $B \leqslant 0_{F M}$. Then by Theorem 2 there exists $x \in F$ such that $x B=0_{M}$. So, $B \leqslant\left(0_{M}: x\right)$. Hence, $B \leqslant$ $A=\vee\left\{\left(0_{M}: x\right) \mid x \in F\right\}$. Therefore, $0_{F M} \leqslant \vee\left\{\left(0_{M}: x\right) \mid x \in\right.$ $F\}=A$. Let $B$ be a compact element such that $B \leqslant A$. Then, there exist $x_{1}, x_{2}, \ldots, x_{n} \in F$ such that $B \leqslant\left(0_{M}: x_{1}\right) \vee\left(0_{M}\right.$ : $\left.x_{2}\right) \vee \cdots \vee\left(0_{M}: x_{n}\right)$. By compactness of $B$, there exist compact elements $Y_{1}, Y_{2}, \ldots, Y_{n} \in M$ such that $x_{i} Y_{i}=0_{M}$ and $B \leqslant$ $Y_{1} \vee Y_{2} \vee \cdots \vee Y_{n}$. As $F$ is a filter, we have $x=x_{1} x_{2} \cdots x_{n} \in F$. Hence, $x B \leqslant x\left(Y_{1} \vee Y_{2} \vee \cdots \vee Y_{n}\right) \leqslant x_{1} Y_{1} \vee x_{2} Y_{2} \cdots \vee x_{n} Y_{n}=0_{M}$. Thus, $B \leqslant 0_{F M}$. This shows that $0_{F M}=\vee\left\{\left(0_{M}: x\right) \mid x \in F\right\}$.

Theorem 4. For $F_{1}, F_{2} \in F\left(L_{*}\right)$

(1) $F_{1} \subseteq F_{2}$ implies $0_{F_{1} M} \leqslant 0_{F_{2} M}$;

(2) $0_{F_{1} M} \wedge 0_{F_{2} M}=0_{\left(F_{1} \cap F_{2}\right) M}$.

Proof. (1) Let $F_{1} \subseteq F_{2}$ and let $X$ be a compact element of $M$ such that $X \leqslant 0_{F_{1} M}$. Then by Theorem $2 s X=0_{M}$, for some $s \in F_{1}$. By assumption, $s \in F_{2}, s X=0_{M}$. Hence, $X \leqslant 0_{F_{2} M}$ and $0_{F_{1} M} \leqslant 0_{F_{2} M}$.

(2) We have $F_{1} \cap F_{2} \subseteq F_{1}, F_{2}$. Hence by $1,0_{\left(F_{1} \cap F_{2}\right) M} \leqslant$ $0_{F_{1} M}, 0_{F_{2} M}$. Thus, $0_{\left(F_{1} \cap F_{2}\right) M} \leqslant 0_{F_{1} M} \wedge 0_{F_{2} M}$. Let $X$ be a compact element in $M$ such that $X \leqslant 0_{F_{1} M} \wedge 0_{F_{2} M}$. Then $X \leqslant 0_{F_{1} M}$ and $X \leqslant 0_{F_{2} M}$. By Theorem 2, there exist elements $s \in F_{1}$ and $r \in F_{2}$ such that $s X=0_{M}$ and $r X=0_{M}$. So $X \leqslant\left(0_{M}: s\right)$ and $X \leqslant\left(0_{M}: r\right)$. Consequently, $X \leqslant\left(0_{M}: s\right) \wedge\left(0_{M}: r\right)=$ $0_{M}:(s \vee r)$ [3]. As $r$ and $s$ are compact elements, $s \vee r$ is also compact. As $F_{1}$ and $F_{2}$ are filters, $s \vee r \in F_{1}$ and $s \vee r \in F_{2}$. This implies that $s \vee r \in F_{1} \cap F_{2}$. Thus, $X \leqslant 0_{M}:(s \vee r)$ where $s \vee r \in F_{1} \cap F_{2}$. That is, $(s \vee r) X=0_{M}$. Hence, by Theorem 2, $X \leqslant 0_{\left(F_{1} \cap F_{2}\right) M}$. That is, $0_{F_{1} M} \wedge 0_{F_{2} M} \leqslant 0_{\left(F_{1} \cap F_{2}\right) M}$. Thus, $0_{F_{1} M} \wedge 0_{F_{2} M}=0_{\left(F_{1} \cap F_{2}\right) M}$.

\section{Weakly Prime and Weakly Primary Elements}

We introduce the concepts of weakly prime and weakly primary elements in lattice modules. These concepts are generalisations of weakly prime elements in multiplicative lattices [4] and weakly primary ideals [5].

Definition 5. A proper element $N$ of $M$ is said to be weakly prime element if $0 \neq a X \leqslant N$ implies $X \leqslant N$ or $a I_{M} \leqslant N$ for every $a \in L, X \in M$.

Definition 6. A proper element $N$ of $M$ is said to be weakly primary element if $0 \neq a X \leqslant N$ implies $X \leqslant N$ or $a^{n} I_{M} \leqslant N$ for every $a \in L, X \in M$.
Example 7. Let $M$ denote a cyclic $Z$-module $Z / 6 Z$ and let $L=L(Z)$ denote the set of all ideals of $Z$ and let $L(Z / 6 Z)$ denote the set of all submodules of $Z / 6 Z$. Then $L(Z / 6 Z)$ is a lattice module over $L$. Let $N=\{0\}$. Then $N$ is a weakly prime element of $M$.

Example 8. Let $R=Z$ denote the set of integers and let $L(R)$ denote the set of all ideals of $R$. Then $L(R)$ is a multiplicative lattice. Let $M=Z \times Z$. Then $M$ is a module over $Z$. Let $L(M)$ denote the set of all submodules of $M$. Then $L(M)$ is a lattice module over $L(R)$. Let $L=(4,0) Z+(0,1) Z$; then $L$ is a weakly primary element of $L(M)$; however it is not weakly prime element of $L(M)$, because $0 \neq(2)(2,1) \subseteq L$. But neither (2) $M \subseteq L$ nor $(2,1) \in L$; see [6].

The next result gives the relation between weakly primary element of $M$ and weakly primary element of $L$.

The study of such concepts for submodules is carried out by Eid [6].

Theorem 9. Let $L$ be a multiplicative lattice, $M$ a lattice module over $L$, and $N$ a weakly primary element of $M$. Then $(N$ : $\left.I_{M}\right)$ is a weakly primary element of $L$.

Proof. Suppose $N$ is a weakly primary element of $M$. Let $0 \neq$ $a b \leqslant\left(N: I_{M}\right)$ and $a \nless\left(N: I_{M}\right)$ where $a, b \in L$. This implies that $a I_{M} \nless N$. But $N$ is a weakly primary element and $0 \neq a b I_{M} \leqslant N$ and $a I_{M} \nless N$. So $b^{n} I_{M} \leqslant N$ for some $n \in Z_{+}$; that is, $b^{n} \leqslant\left(N: I_{M}\right)$. Hence, $\left(N: I_{M}\right)$ is a weakly primary element of $L$.

Now we obtain the relation between weakly primary element of $M$ and weakly prime element of $L$ where $L$ is assumed to be without divisors of zero.

Theorem 10. Let $L$ be a multiplicative lattice and $M$ a lattice module over $L$ and suppose $N$ is a weakly primary element of $M$. Then $\sqrt{\left(N: I_{M}\right)}$ is a weakly prime element of $L$.

Proof. Suppose $N$ is a weakly primary element of $M$. Then by Theorem $9\left(N: I_{M}\right)$ is a weakly primary element of $L$. Let $0 \neq a b \leqslant \sqrt{\left(N: I_{M}\right)}$ with $a \nless \sqrt{\left(N: I_{M}\right)}$. Hence $a^{n} \nless(N$ : $I_{M}$ ) for any $n \in Z_{+}$. That is, $a^{n} I_{M} \nless N$ for any $n \in Z_{+}$. We have $0 \neq(a b)^{k} \leqslant\left(N: I_{M}\right)$ for some $k \in Z_{+}$. That is, $0 \neq a^{k} b^{k} I_{M} \leqslant N$ where $a^{k} I_{M} \nless N$ and $N$ is a weakly primary element $M$. Hence, $\left(b^{k}\right)^{l} I_{M} \leqslant N$ for some $l \in Z_{+}$. That is, $b^{m} I_{M} \leqslant N$ where $k l=m \in Z_{+}$. Thus $\sqrt{\left(N: I_{M}\right)}$ is a weakly prime element of $L$.

\section{Conflict of Interests}

The authors declare that there is no conflict of interests regarding the publication of this paper.

\section{References}

[1] A. Khouja-Al Eaman, "Maximal elements and prime elements in lattice modules," Damascus university Journal for Basic Sciences, vol. 19, no. 2, pp. 9-20, 2003. 
[2] D. D. Anderson, C. Jayaram, and P. A. Phiri, "Baer lattices," Acta Scientiarum Mathematicarum, vol. 59, no. 1-2, pp. 61-74, 1994.

[3] J. A. Johnson, "A-adic completions of Noetherian lattice modules," Fundamenta Mathematicae, vol. 66, pp. 341-371, 1970.

[4] F. Callialp, C. Jayaram, and U. Tekir, "Weakly prime elements in multiplicative lattices," Communications in Algebra, vol. 40, no. 8, pp. 2825-2840, 2012.

[5] S. E. Atani and F. Farzalipour, "On weakly primary ideals," Georgian Mathematical Journal, vol. 12, no. 3, pp. 423-429, 2005.

[6] A. A. Eid, "On weakly primary submodules," Journal of Al Azhar University-Gaza (Natural Sciences), vol. 13, pp. 31-40, 2011. 


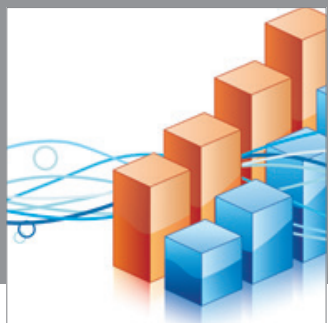

Advances in

Operations Research

mansans

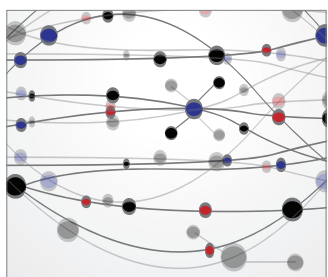

The Scientific World Journal
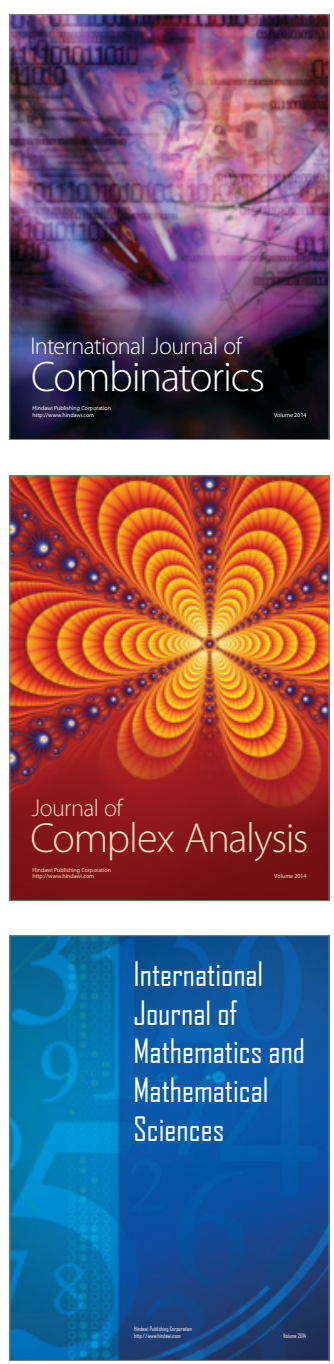
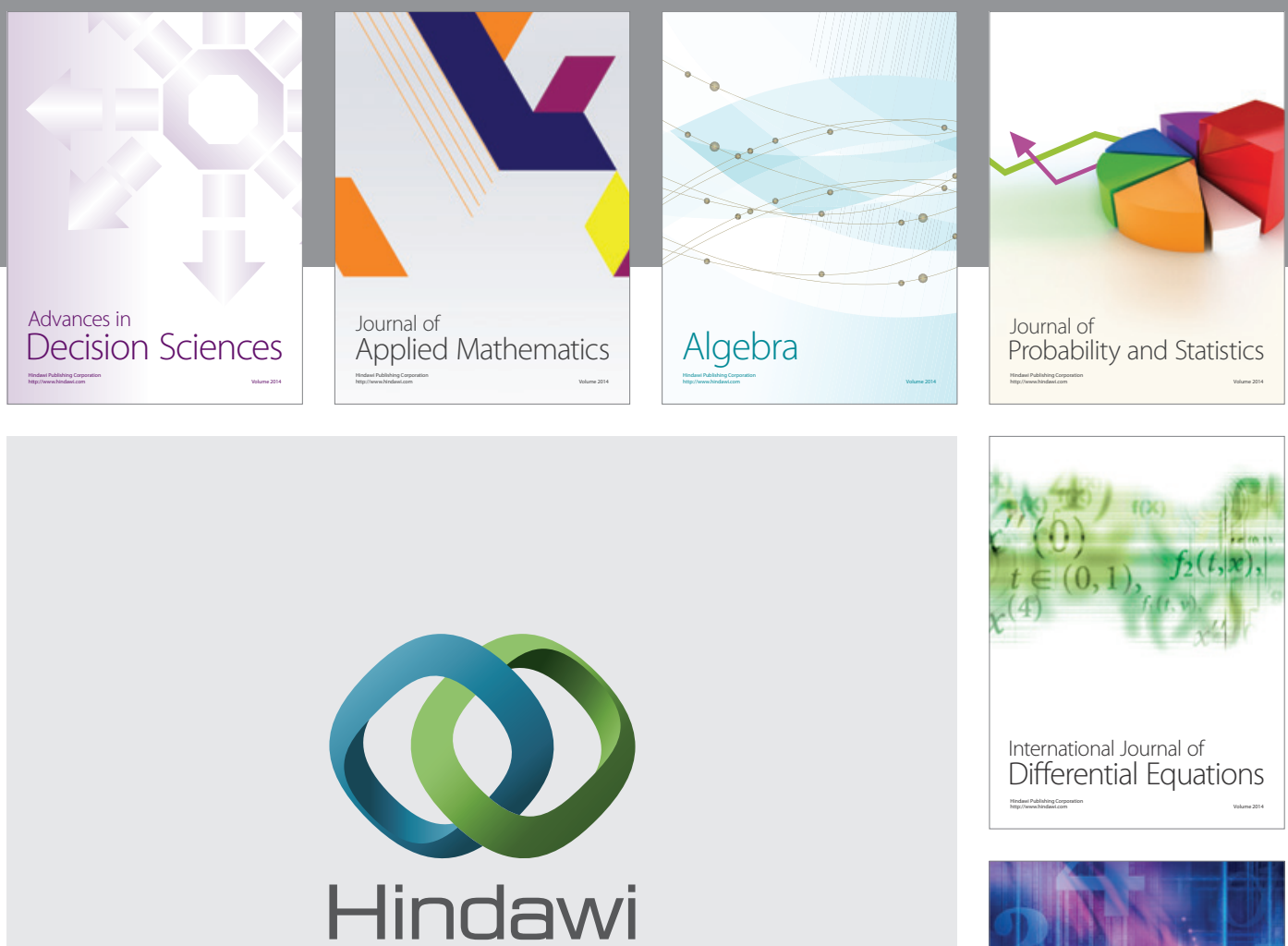

Submit your manuscripts at http://www.hindawi.com
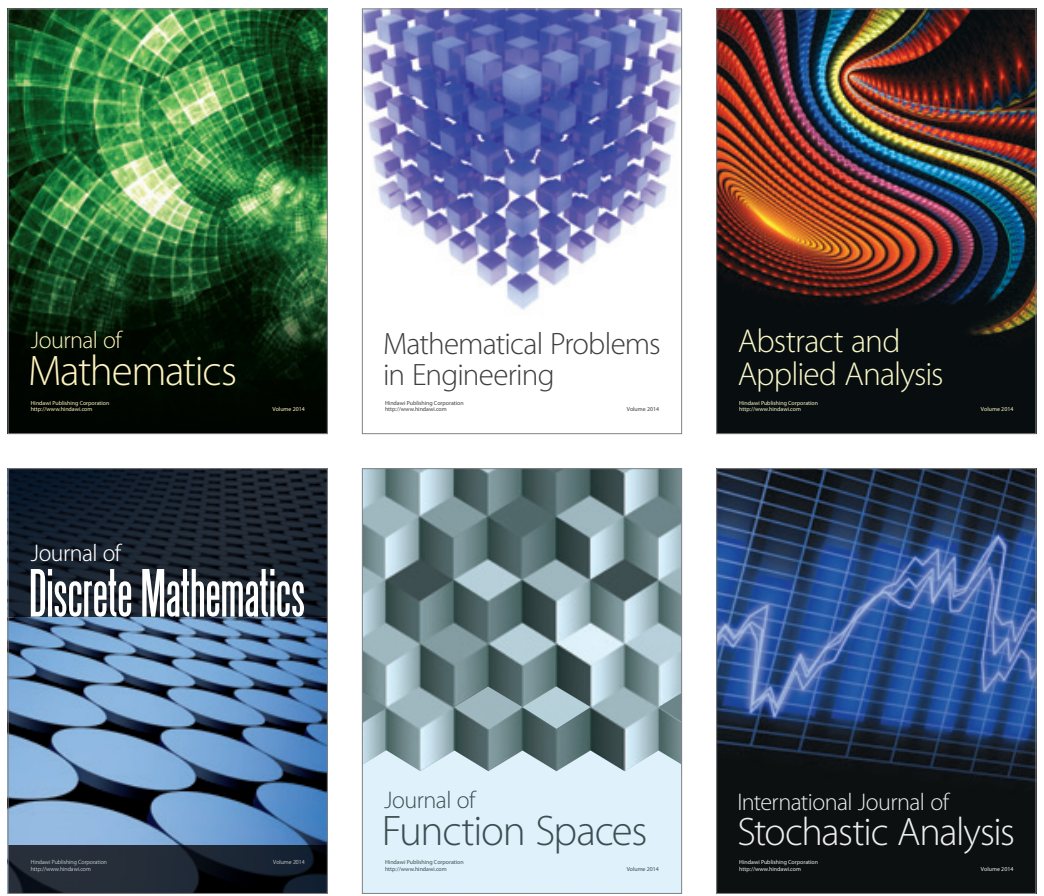

Journal of

Function Spaces

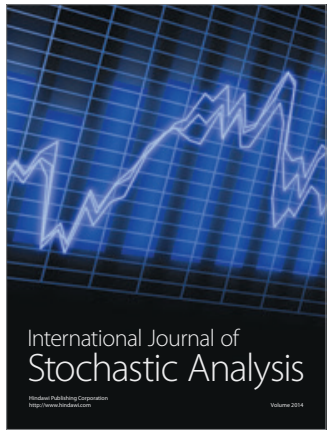

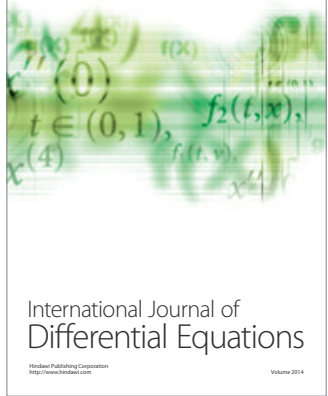
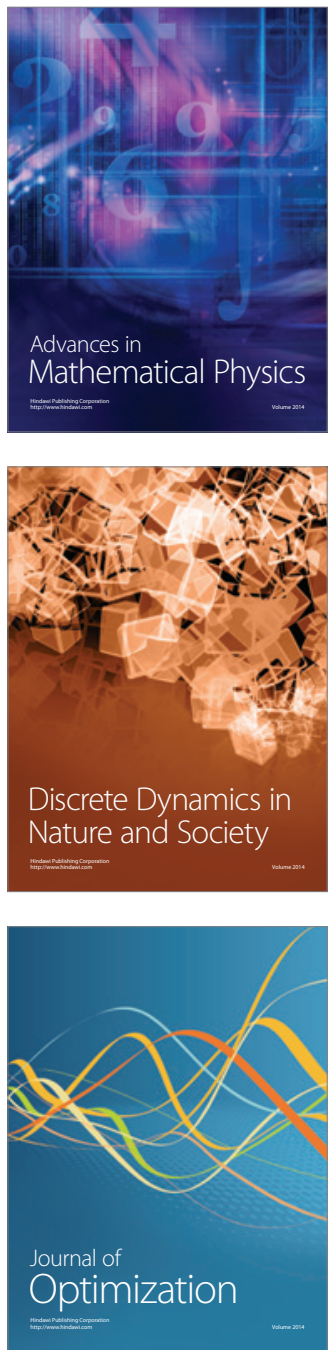